\title{
CARACTERIZAÇÃO DOS RESÍDUOS SÓLIDOS GERADOS EM LATICÍNIOS
}

\section{Characterization of solid waste generated by the dairy industry}

\author{
Antônio Iranaldo Nunes Leite ${ }^{1}$, Rayane Campos Alves ${ }^{2}$, Fernanda Damasceno Soares ${ }^{3}$, \\ Marcelo Henrique Otenio ${ }^{4 *}$, Vanessa Romário de Paula
}

\begin{abstract}
RESUMO
A conscientização ambiental está cada vez mais inserida nas escolhas do consumidor. A preocupação em atender essa demanda tem impulsionado as empresas a buscarem adequação dos seus processos e rotinas. Este estudo tem como objetivo realizar um levantamento dos resíduos sólidos gerados nos laticínios e os respectivos tratamentos e a disposição final. Foram convidados a participar vinte e duas (22) indústrias de diversas regiões do Brasil, classificados quanto ao porte e potencial poluidor. Participaram 7 (sete) laticínios localizados na região Sudeste, 3 (três) na região Sul, 3 (três) na região Centro-Oeste, 3 (três) na região Norte, 7 (sete) na região Nordeste. Foi elaborado um questionário eletrônico estruturado de acordo com a divisão de classe dos resíduos: comuns, químicos e biológicos, e enviado aos laticínios. As respostas demonstraram que as indústrias de laticínios, independente do volume de leite processado com características intrínsecas, frequência e volume gerado não possuem em suas rotinas o gerenciamento dos resíduos. Este estudo demonstra a situação atual de como os laticínios tratam este tema e destaca a necessidade dessas indústrias sem se adaptar às questões ambientais. Baseado na legislação vigente, de resíduos sólidos, a indústria de laticínio deve buscar adequação e inserção de tecnologias sustentáveis em seus processos e rotinas. A adoção dessas práticas tem influência direta e positiva no marketing e comercialização da empresa.

Palavras-chave: gestão ambiental; indústria láctea; produção mais limpa; sustentabilidade.
\end{abstract}

1 Instituto Federal de Educação, Ciência e Tecnologia do Rio Grande do Norte, Currais Novos, RN, Brasil.

2 Centro Universitário Atenas, Paracatu, MG, Brasil.

3 Mestrado Profissional em Ciência e Tecnologia de Leite e Derivados, Juiz de Fora, MG, Brasil.

4 Embrapa Gado de Leite, Av. Eugênio do Nascimento, 610, 36038-330, Aeroporto, Juiz de Fora, MG, Brasil. E-mail: marcelo.otenio@embrapa.br

* Autor para correspondência. 


\begin{abstract}
Environmental awareness is increasingly inserted in the consumer's choices. The concern to meet this demand has driven companies to seek the adequacy of their processes and routines. This study aims to carry out a survey of the solid residues generated in the dairy industry and the respective treatments and the final disposal. Twenty-two (22) industries from different regions of Brazil were invited to participate, classified according to size and potential pollutant. Seven (7) dairy industries were located in the Southeast, 3 (three) in the South, 3 (three) in the Midwest, 3 (three) in the North, 7 (seven) in the Northeast. A structured electronic questionnaire was elaborated according to the class division of the residues: common, chemical and biological, and sent to the dairy. The answers showed that the dairy industries, regardless of the volume of processed milk with intrinsic characteristics, frequency and volume generated, do not have residue management in their routines. This study demonstrates the current state of how dairy industries address this issue and highlight the need for these industries to adapt to environmental issues. Based on current solid waste legislation, the dairy industry should seek to adapt and integrate sustainable technologies into its processes and routines. The adoption of these practices has a direct and positive influence on the marketing and commercialization of the company.
\end{abstract}

Keywords: environmental management; dairy industry; cleaner production; sustainability.

\section{INTRODUÇÃO}

A indústria de laticínios representa uma atividade de grande importância na economia mundial e brasileira. De acordo com dados da Embrapa (2014), o Brasil já é o sexto maior produtor mundial de leite e pode se tornar um dos maiores exportadores de produtos lácteos.

Atualmente os consumidores estão cada vez mais exigentes e ambientalmente conscientes. A necessidade crescente pela preservação dos recursos naturais leva a um movimento progressista de conscientização da sociedade no sentido de cada vez mais, consumir produtos e serviços que gerem menor impacto ambiental, exigindo uma adequação por parte das empresas / indústrias (SILVEIRA et al., 2013).

As questões ambientais têm sido uma preocupação para qualquer empresa. Não só para atender as exigências da legislação, mas por tratar de uma tendência do mercado consumidor. Sendo assim, a responsabilidade socioambiental tornou-se parte fundamental do planejamento de empresas, nos quais a adequação de suas práticas para atender a legislação é fundamental e a consciência passa ser a chave de acesso ao marketing da lucratividade (OLIVEIRA; SERRA, 2010).

A competitividade é motivadora para o desempenho ambiental das empresas. É difícil uma empresa se manter no mercado, focando apenas em seus produtos e serviços, é fundamental apresentar uma imagem perante a sociedade e consumidores, numa comunicação assertiva da atuação em gestão ambiental dos processos da indústria (TATE et al., 2010).

Hoje, uma empresa competitiva deve ser sustentável. Para Delai; Takahashi (2013), uma empresa passa a ser considerada sustentável quando não apenas gera economia, na forma de crescimento, desenvolvimento e eficiência, mas traz também benefícios para 
a área social e para o ambiente, trabalhando para a integridade dos ecossistemas e para a conservação dos recursos da natureza e da biodiversidade. Devido à relevância que o tema tem ocupado na atualidade, vem aumentando o número de empresas brasileiras interessadas em desenvolver práticas que reduzam os impactos que suas atividades causam ao meio ambiente.

Entretanto, observa-se também que estas mesmas empresas não apresentam conhecimento suficiente em gestão ambiental o que é um entrave para alcançarem um grau de eco eficiência adequado, mostrando que um dos grandes impasses da questão é a educação (TELES et al., 2015). A educação ambiental empresarial, melhora a imagem da empresa, e traz benefícios tanto para a própria empresa como para a sociedade, tais como a prevenção da poluição e práticas de produção mais limpa; reestruturação de processos e produtos aliando a redução, a reutilização e a reciclagem, enfatizando na conservação dos recursos naturais (JONES, 2010). O alcance desses objetivos é uma meta para as indústrias, incluindo os laticínios, conscientes de sua responsabilidade socioambiental.

Para Barbieri (2010), a preocupação com o meio ambiente não é recente, mas foram nas últimas três décadas do século XX que ela entrou definitivamente na agenda dos governos de muitos países e de diversos segmentos.

As indústrias de um modo geral são grandes geradoras de resíduos em termos de volume e periculosidade. Dentre as indústrias, estão as de laticínios que geram em seus processos resíduos sólidos, líquidos e gasosos. A gestão adequada destes resíduos deve considerar os seguintes aspectos: prevenção, minimização, reciclagem, tratamento e disposição. O foco desse estudo são os resíduos sólidos.

Apesar da existência de legislação vigente sobre gerenciamento dos resíduos sóli- dos e a responsabilidade dos geradores, verifica-se quase uma ausência de fiscalização pelos órgãos responsáveis, e o descarte inadequado leva estas indústrias a contribuírem com a emissão de resíduos poluentes são meio ambiente.

Segundo Cerati; Lazarini (2009), a consciência ambiental é despertada por meio da Educação Ambiental, que tem como desafio promover a mudança de valores, posturas e atitudes, onde a compreensão é obtida em médios e longos prazos.

Para os efeitos desta Norma NBR 10.004 (ABNT, 2004), são considerados resíduos sólidos, os resíduos com características físicas sólidas propriamente ditas e os líquidos cujas particularidades tornem impróprios para o seu lançamento na rede pública de esgotos, sem o tratamento prévio, para que seja possível seu lançamento em corpos hídricos (CONAMA, 2011).

O objetivo do estudo foi caracterizar os resíduos sólidos gerados nas indústrias de laticínios, buscando identificar as práticas usadas de segregação e a disposição final dada a estes resíduos.

\section{MATERIAL E MÉTODOS}

Para o levantamento dos dados foi usado um questionário on-line utilizando-se a plataforma virtual Survey Monkey ${ }^{\circledR}$. O link do questionário foi encaminhado ao responsável pelo laboratório de controle de qualidade dos laticínios participantes da pesquisa. A natureza da pesquisa foi exploratório-descritiva. O questionário foi encaminhado a 22 laticínios distribuídos nos 5 (cinco) estados brasileiros de diferentes portes. Estes laticínios foram classificados, para este estudo, conforme uma adaptação feita a partir da DN $n^{\circ}$ 217(COPAM, 2017), que regulamenta o licenciamento ambiental, em que considera o porte do empreendimento e o potencial poluidor nos critérios de avaliação nos requisitos legais 
para obtenção do licenciamento ambiental do mesmo. Esta legislação serviu de base para o trabalho, visto que, para conseguir comparar os empreendimentos optou-se por fazer uma adaptação à legislação ambiental do estado de Minas Gerais que é considerada uma das mais avançadas entre os estados participantes. A partir da adaptação da normativa para classificar os empreendimentos obtiveram-se as seguintes classificações: os laticínios de pequeno potencial poluidor foram considerados com recebimento de leite inferior a $15.000 \mathrm{Ld}^{-1}$. Os laticínios de médio potencial poluidor foram considerados com recebimento de leite menor ou igual a $80000 \mathrm{Ld}^{-1}$. Os laticínios de grande potencial poluidor foram considerados com recebimento de leite maior do que $80000 \mathrm{~L} \mathrm{~d}^{-1}$.

Os 22 laticínios foram assim divididos: na região Sudeste: 1 laticínio pequeno, 2 laticínios médios e 4 laticínios grandes, na região Sul: 1 laticínio médio e 2 laticínios grandes, na região Centro-Oeste: 1 laticínio médio e 2 laticínios grandes, na região Norte: 1 laticínio médio e 1 laticínio grande, na região Nordeste: 4 laticínios pequenos, 2 laticínios médios e 1 laticínio grande. Os representantes dos laticínios foram convidados a participar por um e-mail enviado pelo responsável pela pesquisa e que continha um link de acesso para o preenchimento do questionário, a primeira pergunta constava do termo de consentimento e, após aceitar participar, as demais perguntas deveriam ser respondidas na ordem como apresentado na Figura 1.

Dos 22 laticínios selecionados e convidados para participar da pesquisa após 1 mês, 12 deles responderam completamente o questionário. Assim a adesão a pesquisa foi de mais de $54 \%$.

Pelo retorno obtido e com as características das respostas optou-se por tabular os resultados de forma aberta e ampla, comparando-se as respostas para verificar as rotinas e práticas dos respondentes no manejo de resíduos gerados nos laticínios.

\section{RESULTADOS E DISCUSSÃO}

As respostas foram trabalhadas conforme divisão de classes de resíduos, como abordado no questionário enviado: resíduos comuns, resíduos químicos e resíduos biológicos gerados.

Para resíduos comuns, a primeira pergunta foi sobre a existência de plano de gerenciamento de resíduos, com intuito de verificar se há algum tipo de controle quanto à geração, segregação e destinação dos resíduos gerados nos laticínios. Dos respondentes pouco mais de $41 \%$ responderam sim nesta questão. A questão ambiental para indústrias de laticínios deveria ser um ponto mais considerado.

Estes resultados estão em conformidade com resultados encontrados por Jerônimo et al. (2012) que afirma haver grande carência em dados quantitativos e qualitativos. Afirma ainda que muitas indústrias adotam soluções cômodas e simples para o problema da deposição final destes resíduos, sem critérios técnicos que podem significar agressões ao meio ambiente.

O gerenciamento de resíduos tem papel importante neste contexto, pois parte dos impactos ambientais é causada pelo descarte inadequado de resíduos. Ainda neste sentido, Jerônimo et al. (2012) afirma que podem existir diferenças no destino dado aos resíduos produzidos pelas indústrias de laticínios, quando se compara empresas situadas em áreas urbanas e empresas em áreas rurais. Neste sentido, no Brasil, podemos encontrar diferenças também entre regiões, onde as mais desenvolvidas e com maior acesso a informação apresentam melhores indicadores no gerenciamento de resíduos.

Dentro dessa abordagem, quando questionados sobre coleta seletiva, mais de $83 \%$ dos laticínios responderam que possuem coleta seletiva para os resíduos gerados. Entretanto apenas $20 \%$ dessas indústrias realizam o gerenciamento desses resíduos, ou seja, 80\% fazem a separação dos resíduos, porém não 


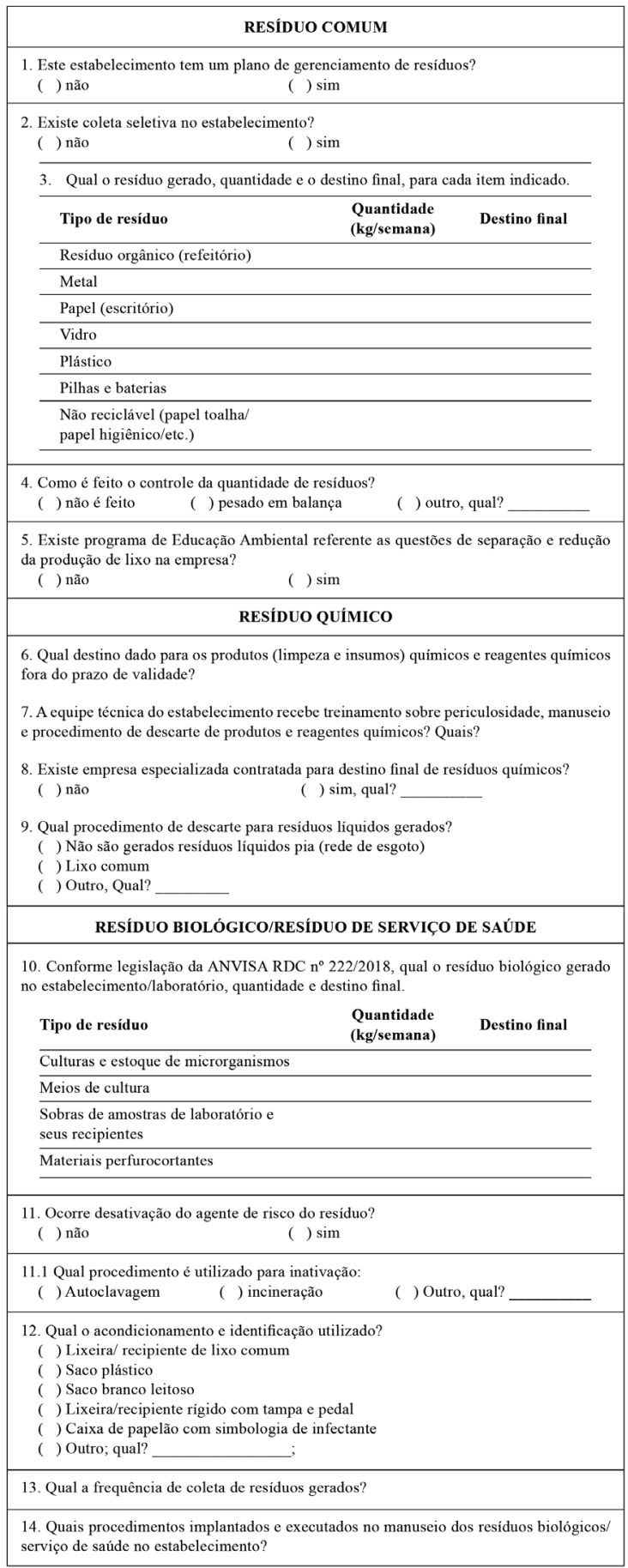

Figura 1 - Levantamento sobre resíduos na cadeia produtiva do leite.

Fonte: do autor. 
fazem nenhum controle de quantificação e destinação final. Este percentual de $20 \%$, na amostragem total refere-se a grandes plantas industriais, que pelas suas características e sua inserção no mercado são mais cobradas para esta abordagem. Ainda com as respostas obtidas, verificou-se que os laticínios, que foram classificados como grandes poluidores conforme a adaptação da normativa, apresentam ações de gerenciamento de resíduos e coleta seletiva implantada. Isto ocorre devido ao amadurecimento gerencial e de marketing mais exigentes e atuantes em grandes empresas e, pela grande geração de resíduos, necessitando de mais atenção. Neste ponto, os médios e pequenos laticínios, na ausência de processos mais desenvolvidos tanto gerencial quanto de relação produto e mercado, além do pequeno volume de resíduos gerados, desprezam a necessidade de controle, e quantificação dos resíduos segregados na coleta seletiva que dizem realizar.

De acordo com dados da ABRELPE (2014), a geração de resíduo sólidos no Brasil, de 2013 para 2014 , cresceu $2,9 \%$ e foi de aproximadamente 78,6 milhões de toneladas, enquanto que a destinação final inadequada deste resíduo continuou praticamente igual, neste mesmo período, em termos percentuais, caindo apenas $0,1 \%$. Isso demonstra que a educação ambiental e a gestão estratégica de resíduos ainda têm um longo percurso a percorrer, especialmente no Brasil. Embora, as práticas ambientais devem ser vistas como parte das responsabilidades sociais das empresas, não podendo ser relevadas em segundo plano, já que são vistas também como estratégia de competição (SILVEIRA et al., 2013).

$\mathrm{Na}$ terceira pergunta foi solicitada a informação de quantidade gerada de cada tipo de resíduo e a destinação final dada. Para essa pergunta, 75\% dos laticínios informaram as quantidades de resíduos geradas. Até mesmo aqueles que anteriormente responderam não possuir gerenciamento apresentaram uma estimativa. Esta resposta foi dada certamente porque os laticínios são sensíveis para a questão, e conseguem vislumbrar a necessidade de acompanhar os processos geradores de resíduos e neste ponto poderia ser inserida a temática dos 3R's (reduzir, reutilizar e reciclar). Em 58\% dos laticínios, os resíduos recicláveis têm uma destinação final adequada.

Silveira et al. (2013) afirma que a importância da implantação de sistema de gestão ambiental é cada vez mais necessária e traz inúmeros benefícios a indústria. $\mathrm{Na}$ indústria de laticínios, estes benefícios podem ser traduzidos como redução dos custos de produção, economia de água entre outros, demonstrando a necessidade cada vez maior da implantação de um processo de gestão ambiental neste segmento. Outro benefício gerado seria o fator diferencial no mercado, levando a empresa a estar sempre em processo de melhoria continua.

A pergunta sobre a existência de programa de Educação Ambiental foi negativa para mais de $66 \%$ dos laticínios, o que é contraditório frente às respostas dadas na questão anterior. A educação ambiental é uma das premissas da gestão ambiental. As ações para a segregação dos resíduos por classes iniciamse com a conscientização e mobilização dos envolvidos no processo, o que fica caracterizado como educação ambiental. Para Viveiros et al. (2015), educação ambiental promove uma abordagem multidisciplinar, para que as gerações futuras desenvolvam uma nova relação com o ambiente.

A segunda etapa do questionário abordou o tratamento dado aos resíduos químicos. As respostas da primeira pergunta sobre a destinação de produtos fora da validade mostrou que $41 \%$ destinam adequadamente esses resíduos, devolvendo para o fornecedor ou contratando empresa especializada. Outros $41 \%$ tratam esse resíduo e descartam na rede de esgoto, o que pode ser extremante prejudicial para as Estações de Tratamento de Esgoto (ETE) dependendo do resíduo.

Apesar das dificuldades dos pequenos 
e médios laticínios, em obter o licenciamento ambiental, $75 \%$ dos laticínios pesquisados se mostram preocupados com o manuseio e procedimentos de descarte dos produtos e reagentes químicos. Estes resultados obtidos podem ser justificados devido às indústrias oferecerem treinamentos a suas equipes técnicas. Os treinamentos quando são realizados abordam todo o ciclo de vida do produto químico, até a destinação final adequada, minimizando riscos de impacto ambiental.

A utilização de empresa especializada contratada para destinar os resíduos químicos não é comum nos laticínios, pois $67 \%$ dos respondentes informaram descartar os resíduos líquidos na pia. O desconhecimento dos componentes químicos dos resíduos gerados nas análises laboratoriais é comum nos laticínios, assim como, os riscos que os mesmos podem causar ao meio ambiente, fazem com que seu descarte não seja tratado com o critério necessário e são direcionados para as ETE ou rede de esgoto pública.

Sabe-se que, há algumas décadas, as medidas de proteção e regulação ambiental eram obstáculos para a competitividade das organizações devido aos caros investimentos. Hoje em dia, a questão ambiental mudou e a mesma já possui lugar de destaque nos debates nacionais e internacionais. Diante dessa mudança, empresas vêm sendo obrigadas a reduzirem os impactos causados ao meio ambiente e até mesmo a recuperarem os danos ambientais decorrentes de suas atividades (STEVENS et al., 2012). A legislação, o mercado e a menor disponibilidade de recursos naturais têm pressionado as mesmas a mudarem a forma de produzir e de extrair matéria prima da natureza (BARBIERI et al., 2010). Sendo assim, a principal regra a ser adotada para o gerenciamento dos resíduos é a da responsabilidade objetiva, onde, quem gera o resíduo torna-se responsável pelo mesmo, e o cuidado com o descarte dos insumos acima de tudo será um compromisso com a sociedade. As empresas que atuam de forma ambientalmente responsável, além de ser uma solução eficaz, irá agregar valor na própria imagem da junto à sociedade onde será considerada ecologicamente correta (MENDES et al., 2015).

Os resíduos biológicos foram abordados na terceira etapa do questionário. As respostas quanto à geração, segregação e destinação desses resíduos demonstraram que os laticínios destinam os resíduos biológicos corretamente, em mais de 84\%. Laticínios de pequeno porte, que representaram $16 \%$ dessas respostas, utilizam material descartável para análises microbiológicas e utilizam a fornalha da caldeira como forma de inativação biológica e destinação final do resíduo. Esse procedimento pode prejudicar o funcionamento do equipamento, além de ser uma forma de descarte inadequado, que pode causar sérios danos ao meio ambiente e a saúde humana, pois alguns resíduos contêm gás poluente que pode causar vários tipos de doenças e reações em plantas (STEVENS et al., 2012).

Dos laticínios respondentes que geram resíduos biológicos, 84\% informaram que realizam a inativação dos agentes de risco através do processo de auto clavagem. Após esse procedimento os resíduos são descartados na pia, já que não apresentam mais risco biológico. As sobras de amostras de produtos analisados somente considerados resíduos biológicos quando apresentarem resultados microbiológicos fora dos padrões estabelecidos pela legislação pertinente. Pois caso contrário, são produtos próprios para consumo.

A pergunta sobre as formas de acondicionamento dos resíduos indicou que $100 \%$ dos respondentes não usam recipientes adequados para o descarte dos resíduos, são usadas lixeiras sem identificação e sacos comuns. Uma situação que contraria a informação de implantação de coleta seletiva, que necessita de coletores individualizados para cada classe de resíduo.

Em relação aos procedimentos de manuseio dos resíduos biológicos, $50 \%$ dos 
laticínios respondentes informaram ter implantados, em algum nível em suas atividades laboratoriais. E pouco mais de 30\% desses informaram sobre a elaboração de Procedimentos Operacionais Padrão - POP e a implantação de Boas Práticas Laboratoriais - BPL, visto que no ambiente interno, as organizações precisam que as práticas implementadas sejam cumpridas de forma eficiente. Para isto é necessária a implantação de um sistema de gerenciamento ambiental sistêmico dentro da rotina da empresa, estabelecendo intercâmbio de informações entre atores internos e externos e determinando requisitos legais para estas práticas. Entretanto, no Brasil, a gestão ambiental tem se caracterizado desarticulada, sem coordenação, e sempre com escassez de recursos (MARCKMANN, 2012).

Baseado na Política Nacional de Resíduos Sólidos (BRASIL, 2010), é importante a adequação das indústrias de laticínios quanto ao manejo dos resíduos gerados, principalmente na perspectiva de relacionar seu produto a uma conduta e práticas sustentáveis. Da mesma maneira que atualmente a cadeia produtiva do leite e os laticínios têm mostrado preocupação com questões relacionadas à geração de poluição e tratamento de efluentes gerados, o resíduo sólido deve entrar neste contexto e ser considerado como mais um ponto importante no planejamento e nas atividades destas indústrias.

\section{CONCLUSÕES}

Como em todo sistema de produção, a indústria de laticínio gera resíduos, independente do volume processado. Entretanto sua especificidade, frequência e volume gerado são fatores determinantes para escolha do gerenciamento adequado dos resíduos.

Embora já existam algumas práticas de gerenciamento de resíduos inseridas nas rotinas dos laticínios, não estão sistematizadas num plano de gestão ambiental. As questões levantadas nesse trabalho são úteis para nortear os laticínios no que tange a necessidade de adequação de suas rotinas, buscando alternativas que minimizem os impactos dos seus processos ao meio ambiente.

\section{REFERÊNCIAS}

ABNT. NBR 1004:2004. Resíduos Sólidos Classificação. Rio de Janeiro, 2004.

ASSOCIAÇÃO BRASILEIRA DE EMPRESAS DE LIMPEZA PÚBLICA E RESÍDUOS ESPECIAIS (ABRELPE). Panorama de resíduos sólidos no Brasil. 2014. São Paulo: ABRELPE, 2014.

ANVISA. Resolução RDC n. ${ }^{\circ} 222$, de 28 de março de 2018. Regulamenta as Boas Práticas de Gerenciamento dos Resíduos de Serviços de Saúde e dá outras providências. Diário Oficial da República Federativa do Brasil, Brasília, 29 mar. 2018.

BARBIERI, J. C. et al. Inovação e sustentabilidade: novos modelos e proposições. Revista de Administração de Empresas, v. 50, n. 2, p. 146-154, 2010.

BRASIL. Lei 12.305 , de 02 de agosto de 2010. Institui a Política Nacional de Resíduos Sólidos; altera a Lei $\mathrm{n}^{\circ} 9.605$, de 12 de fevereiro de 1998; e dá outras providências. Diário Oficial da República Federativa do Brasil, Brasília, 03 ago. 2010.

CONAMA. Resolução n ${ }^{\circ} 430$, de 13 de maio de 2011. Dispõe sobre as condições e padrões de lançamento de efluentes, complementa e altera a Resolução $n^{\circ}$ 357, de 17 de março de 2005, do CONAMA. Diário Oficial da República Federativa do Brasil, Brasília, 16 mai. 2011.

COPAM. Deliberação Normativa n. ${ }^{\circ} 217$ de 08 de dezembro de 2017. Estabelece critérios para classificação, segundo o porte e potencial poluidor, bem como os critérios locacionais a serem utilizados para definição 
das modalidades de licenciamento ambiental de empreendimentos e atividades utilizadores de recursos ambientais no Estado de Minas Gerais, e dá outras providências. Minas Gerais, Belo Horizonte, 08 dez. 2017. Diário do Executivo.

CERATI, T. M.; LAZARINI, R. A. M. A pesquisa ação em educação ambiental: uma experiência no entorno de uma unidade de conservação urbana. Ciência \& Educação, v. 15, n. 2, p. 383-392, 2009.

DELAI, I.; TAKAHASHI, S. Corporate sustainability in emerging markets: insights from the practices reported by the Brazilian retailers. Journal of Cleaner Production, v. 47, p. 211-221, 2013.

EMPRESA BRASILEIRA DE PESQUISA AGROPECUÁRIA (EMBRAPA). Visão 2014-2034: o futuro do desenvolvimento tecnológico da agricultura brasileira: síntese. Brasília: Embrapa, 2014. Disponível em: $<$ https://www.embrapa.br/busca-depublicacoes/-/publicacao/987801/visao2014-2034-o-futuro-do-desenvolvimentotecnologico-da-agricultura-brasileira-sintese.> Acesso em: 24 nov. 2017

JERÔNIMO, C. E. et al. A qualidade ambiental e sanitária das indústrias de laticínios do município de Mossoró-RN. Electronic Journal of Management, Education and Environmental Technology (REGET), v. 7, n. 7, p. 1349-1356, 2012.

JONES, C. Exploring new ways of assessing the effect of regulation on environmental management. Journal of Cleaner Production, v. 18, n. 13 , p. 1229-1250, 2010.

MARCKMANN, K. Elaboração de proposta do manual de Gestão Ambiental da UFRGS e estudo de caso de aplicação. 2012. 105 p. Trabalho de conclusão de curso (Graduação em Engenharia Ambiental) - Instituto de pesquisas hidráulicas e escola de engenharia,
Universidade Federal do Rio Grande do Sul, Porto Alegre, 2012.

MENDES, G. S.; SCHREIBER, D.; SILVA, M. O. Inovação e prática ambiental: um estudo de caso em empresas de embalagens do Vale do Sinos, Rio Grande do Sul, Brasil. In: CONGRESSO LATINO AMERICANO DE GESTÃO DA TECNOLOGIA, 16., 2015, Porto Alegre. Anais eletrônicos... Porto Alegre: UFRGS, 2015. Disponível em: <http:// altec2015.nitec.co/altec/papers/458.pdf $>$. Acesso em: 28 mar. 2018.

OLIVEIRA, O. J.; SERRA, J. R. Benefícios e dificuldades da gestão ambiental com base na ISO 14001 em empresas industriais de São Paulo. Produção, v. 20, n. 3, p. 429-438, 2010.

STEVENS, P. A. et al. A critical review of classification of organizations in relation to the voluntary implementation of environmental management systems. Journal of Environmental Management, v. 113, p. 206$212,2012$.

SILVEIRA, M. P; ALVES, J. N.; FLAVIANO, V. Os desafios da implantação de um sistema de gestão ambiental: estudo de caso em uma indústria de laticínios. Revista Gestão \& Sustentabilidade Ambiental, v. 2, n. 2, p. 88106, 2013.

TATE, W. L.; ELLRAM, L. M.; KIRCHOFF, J. O. N. F. Corporate social responsibility reports: a thematic analysis related to supply chain management. Journal of Supply Chain Management, v. 46, n. 1, p. 19-44, 2010.

TELES, C. D. et al. Characterization of the adoption of environmental management practices in large Brazilian companies. Journal of Cleaner Production, v. 86, p. 256 e 264, 2015.

VIVEIROS, E. P. et al. Por uma ética ambiental. Engenharia Sanitária e Ambiental, v. 20, n. 3, p. 331-336, 2015. 